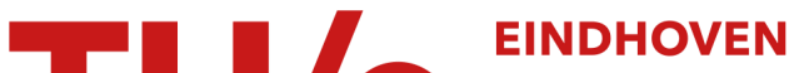 UNIVERSITY OF TECHNOLOGY
}

\section{Complex permittivity measurements with a low cost parabolic resonant cavity}

\section{Citation for published version (APA):}

Federico, G., Reniers, A. C. F., Roc'h, A., Bronckers, L. A., \& Visser, H. J. (2019). Complex permittivity measurements with a low cost parabolic resonant cavity. In Proceedings of the 14th Loughborough Antennas and Propagation Conference (LAPC 2018) [8643262] Institution of Engineering and Technology (IET). https://doi.org/10.1049/cp.2018.1455

DOI:

10.1049/cp.2018.1455

Document status and date:

Published: 18/02/2019

\section{Document Version:}

Accepted manuscript including changes made at the peer-review stage

\section{Please check the document version of this publication:}

- A submitted manuscript is the version of the article upon submission and before peer-review. There can be important differences between the submitted version and the official published version of record. People interested in the research are advised to contact the author for the final version of the publication, or visit the $\mathrm{DOI}$ to the publisher's website.

- The final author version and the galley proof are versions of the publication after peer review.

- The final published version features the final layout of the paper including the volume, issue and page numbers.

Link to publication

\section{General rights}

Copyright and moral rights for the publications made accessible in the public portal are retained by the authors and/or other copyright owners and it is a condition of accessing publications that users recognise and abide by the legal requirements associated with these rights.

- Users may download and print one copy of any publication from the public portal for the purpose of private study or research.

- You may not further distribute the material or use it for any profit-making activity or commercial gain

- You may freely distribute the URL identifying the publication in the public portal.

If the publication is distributed under the terms of Article $25 \mathrm{fa}$ of the Dutch Copyright Act, indicated by the "Taverne" license above, please follow below link for the End User Agreement:

www.tue.nl/taverne

Take down policy

If you believe that this document breaches copyright please contact us at:

openaccess@tue.nl

providing details and we will investigate your claim. 


\title{
Complex Permittivity Measurements with a Low Cost Parabolic Resonant Cavity
}

\author{
G. Federico, A.C.F. Reniers, A. Roc'h, L.A. Bronckers and H.J. Visser \\ Dep. of Electrical Engineering, Eindhoven University of Technology, Eindhoven, the Netherlands \\ g.federico@tue.nl
}

Keywords: Loss tangent, material characterization, open resonator, parabolic cavity, relative permittivity.

\begin{abstract}
A low-cost Fabry Perot open resonator is used to measure the complex permittivity of flat sheets of dielectric materials in the $\mathrm{X}$-band. The method is based on the measurement of the resonant frequency shift of the fundamental mode, described by the Gaussian beam theory. Instead of spherical mirrors, conventionally used for open resonators, the cavity is formed by parabolic reflectors, originally intended for satellite communications, reducing the production costs. A technique to improve the sample alignment is introduced, and to validate the results of the parabolic cavity, the relative permittivity and loss tangent of three different samples are measured.
\end{abstract}

\section{Introduction}

For many applications, knowledge of substrate material properties is key, such as in antenna design or electronic devices design [1]. For dielectric materials, the most important parameters are its relative permittivity $(\varepsilon r)$ and loss tangent (tan $\delta)$, which are often unknown at the frequency of interest or calculated with an unknown uncertainty. Most standard measurement techniques used for characterizing materials are destructive or require an expensive setup. In this paper, a lowcost solution is proposed to extract the relative permittivity and loss tangent in the X-band by using an open cavity resonator. The advantage is that instead of spherical mirrors, which need to be manufactured with high production cost, the cavity is formed by identical parabolic mirrors, generally used for satellite communications and already available on the market at low cost.

Two types of open resonator are conventionally used for material characterization: the hemispherical resonator [2], formed by one spherical mirror and one plane mirror, and confocal resonator, with two spherical mirrors [3]. In the first case, the sample to characterize is placed on top of the planar mirror and, in the second case, in the middle of the cavity. This paper is focused on the use of parabolic mirrors in a confocal configuration. Since the goal is the characterization of solid dielectric materials, the confocal configuration is preferred because it avoids the airgap between the plane mirror and the sample, which causes larger errors proportional to the frequency [4]. Three different samples were used to test the experimental setup: RO4003C, RO4835 and a sample of FR4, whose complex permittivity provided by datasheet was used as reference. A further validation is obtained by using two standard non-resonant methods as comparison: the twomicrostrip line method and the two-layer stripline method [5]. This paper describes, in section two, the setup to obtain the material properties and the theory used to derive the parameters from a measurement. In section three the measurements results are provided and the uncertainty analysis applied. Section four discusses the results obtained and the comparison with respect to the spherical structure. Finally, section five concludes the work.

\section{Theory}

An open resonator can be realized with different geometries, like, for example, hemispherical, concentric or confocal [6]. The most straightforward open resonant cavity is formed by two curved mirrors aligned in front of each other. In all geometries listed above, propagation of the fundamental $\mathrm{TEM}_{0,0, \mathrm{q}}$ mode in the cavity is described using the scalar Gaussian beam theory [7]. It implies that the fundamental mode propagates with a Gaussian intensity profile (as illustrated in Figure 1). Although many higher order TEM modes could be supported inside the cavity, we only consider the resonant frequency of the $\mathrm{TEM}_{0,0, \mathrm{q}}$ mode, which can be calculated for an empty cavity with [8]:

$$
f_{r, e}=\frac{c}{2 D}\left\{q+1+\frac{1}{\pi} \operatorname{arcos}\left(1-\frac{D}{R_{0}}\right)\right\}
$$

where $c$ is the speed of light, $D$ the distance between the reflectors, $R_{0}$ the radius of curvature, and $q$ the axial mode number.

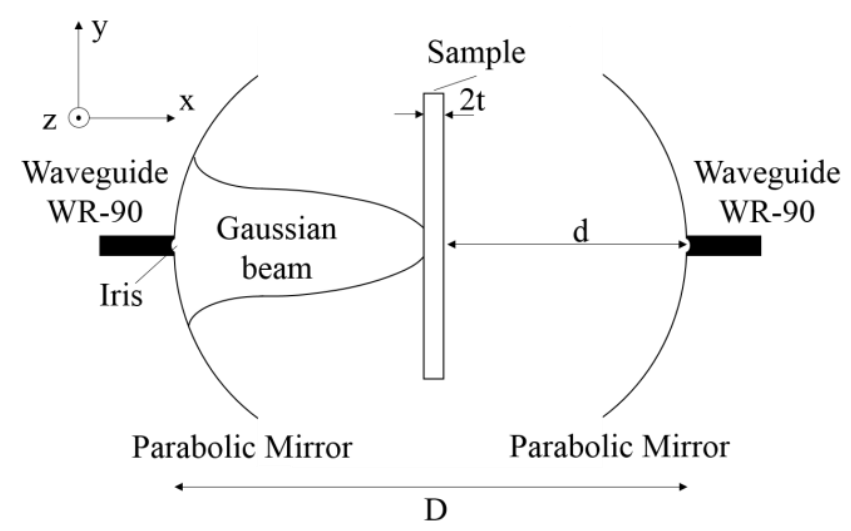

Figure 1. Sketch of the resonant cavity with parabolic dishes. 
When the dielectric sheet of thickness $2 t$ is placed at the center of the cavity, the resonant frequency shifts proportionally to the refractive index $n$. Applying boundary conditions to the discontinuity inside the cavity (air - dielectric) [8], [9], the following transcendental equation is obtained to calculate the refractive index $n$ and, consequentially, for dielectric materials the relative permittivity $\varepsilon_{r}=n^{2}$, depending on whether the mode is symmetric [4], [8]:

$$
\frac{1}{n} \cot \left(n k_{s} t-\Phi_{t}\right)=\tan \left(k_{s} d-\Phi_{d}\right)
$$

or antisymmetric:

$$
\frac{1}{n} \tan \left(n k_{a} t-\Phi_{t}\right)=-\tan \left(k_{a} d-\Phi_{d}\right)
$$

where:

$$
\begin{gathered}
\Phi_{t}=\tan ^{-1}\left(\frac{2 t}{n k w_{0}^{2}}\right), \\
\Phi_{d}=\tan ^{-1}\left\{\frac{2}{k w_{0}^{2}}\left(d+\frac{t}{n^{2}}\right)\right\}-\tan ^{-1}\left(\frac{2 t}{n^{2} k w_{0}^{2}}\right),
\end{gathered}
$$

and:

$$
k w_{0}^{2}=2 \sqrt{\left\{\left(d+\frac{t}{n^{2}}\right)\left(R_{0}-d-\frac{t}{n^{2}}\right)\right\}}
$$

In Equations (2) and (3), $k_{\mathrm{s}}$ and $k_{\mathrm{a}}$ are the free-space phase constant of the symmetric and antisymmetric modes respectively equal to $k=2 \pi f_{r, f} / c$, with $f_{\mathrm{r}, \mathrm{f}}$ the resonant frequency of the filled cavity.

Calculation of the loss tangent is based, instead, on the measurement of the quality factor $Q$, of the empty cavity $Q_{0}$ and the loaded cavity $Q_{1}$. The decrease of the quality factor of the resonator is proportional to the loss tangent of the inserted dielectric material [4] that can be calculated as follows [8]:

where:

$$
\tan \delta=\frac{1}{Q_{e}} \frac{\Delta t+d}{\Delta t+\left(\frac{1}{2 k}\right) \sin 2\left(k d-\Phi_{d}\right)}
$$

$$
\begin{aligned}
& \Delta_{s}=\frac{n^{2}}{n^{2} \sin ^{2}\left(n k_{s} t-\Phi_{t}\right)+\cos ^{2}\left(n k_{s} t-\Phi_{t}\right)} \\
& \Delta_{a}=\frac{n^{2}}{n^{2} \cos ^{2}\left(n k_{a} t-\Phi_{t}\right)+\sin ^{2}\left(n k_{a} t-\Phi_{t}\right)}
\end{aligned}
$$

for symmetric and antisymmetric modes respectively. The quantity $1 / \mathrm{Q}_{\mathrm{e}}$ is equal to:

where:

$$
\frac{1}{Q_{e}}=\frac{1}{Q_{l}}-\frac{1}{Q_{d}}
$$

$$
Q_{d}=Q_{0} \frac{2(t \Delta+d)}{D(\Delta+1)}
$$

is the quality factor for the same cavity, containing a sample with the same thickness and permittivity, but loss-free.

To apply the Gaussian beam approximation, sample dimensions need to be larger than the beam waist of the fundamental mode, whose radius is given by [6]:

$$
w^{2}(x)=w_{0}^{2}\left[1+\left(\frac{\lambda x}{\pi w_{0}^{2}}\right)^{2}\right]
$$

where:

$$
w_{0}^{2}=\frac{2}{k} \sqrt{D\left(R_{0}-D\right)}
$$

is the beam waist radius at $x=0$, considering the origin of the Cartesian coordinate system at the center of the cavity.

The Gaussian beam theory assumes that the equiphase surfaces of the beam are convex. This means that the sample should have a convex shape to match the phase front of the beam instead of the flat shapes of our samples. As shown in [2], this error can be compensated by adding to the thickness the amount $\delta t$ equal to:

$$
\delta t=\frac{w(t)^{2}}{4 R(t)}
$$

Where $w(t)$ and $R(t)$ are respectively the beam radius and the radius of curvature of the phase front at $x=t$.

\section{Measurement setup and Results}

Investigation of the open parabolic resonator has been divided into two parts. Common open resonators are usually designed by using spherical mirrors. Therefore, before proceeding to the realization of setup, the possibility to use parabolic instead of spherical reflectors is investigated first.

\subsection{Simulations}

The software used for the simulations was CST Microwave Studio ${ }^{\circledR}[10]$. Because the simulation model is many times larger than the wavelength, the time-domain solver was considered the most suitable for this purpose.

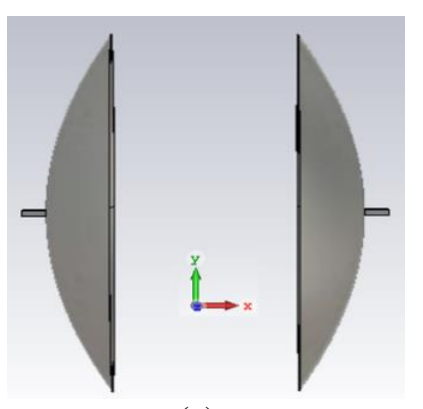

(a)

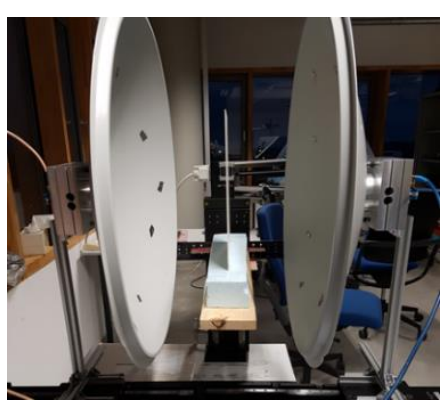

(b)
Figure 2. (a) Image of the cavity in CST and, (b) picture of the setup in laboratory. 


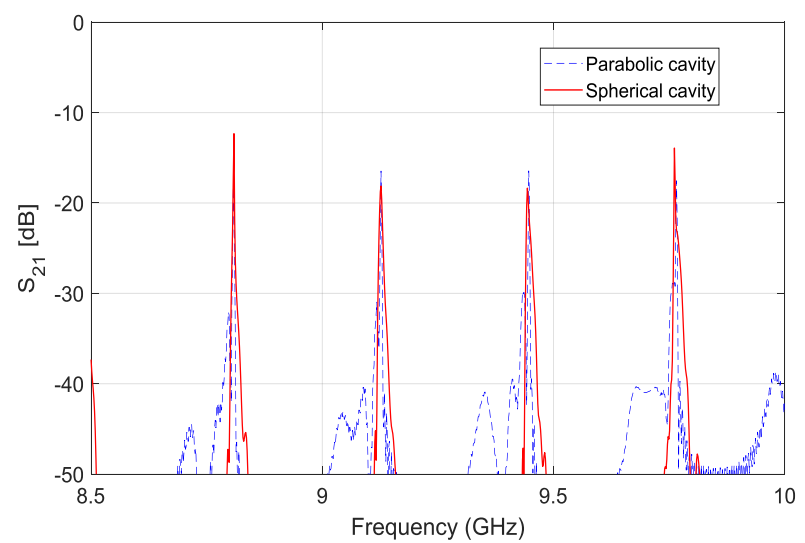

Figure 3. Simulation of the $S_{21}$ : parabolic vs. spherical cavity resonances.

Two empty cavities are simulated first with parabolic reflectors (see Figure 2a) and then an equivalent cavity with spherical reflectors. In the first case, the parabolic reflectors have a curvature approximately described by equation $y=0.01086 x^{2}$ (in $\mathrm{cm}$ ), with a diameter of $60 \mathrm{~cm}$. They are fed by two waveguides WR-90 $(8.2 \mathrm{GHz}-12.4 \mathrm{GHz})$ and excited through an iris of diameter $9.5 \mathrm{~mm}$. A sketch of the simulated setup is shown if Figure 1.

In order to reduce the simulation time, the frequency range is reduced to $8.5-10 \mathrm{GHz}$, enough, however, to study the behavior of the two geometries.

For the simulation of the spherical cavity, an approximate radius of curvature that better follows the parabolic profile is calculated. It results in being $46 \mathrm{~cm}$, approximately two times the focal point of the parabolic reflector. The diameter of the reflectors and the iris are chosen the same as the previous case, equal to $60 \mathrm{~cm}$ and $9.5 \mathrm{~mm}$ respectively. The results of both simulations are displayed in Figure 3. It shows the transmission coefficient $S_{21}$ of the spherical and parabolic cavity zoomed on the range $-15 \mathrm{~dB}$ to $-50 \mathrm{~dB}$. Compared to the measured $S_{21}$, the resonant frequencies are slightly different. This is due to a computational limit for the number of mesh cells. The convergence test performed did not give a definitive number of mesh cells, but, for our purpose, it was enough to establish the similarity of the result between parabolic and spherical reflectors. Since the cavities are simulated in their confocal region (distance equal to the radius of curvature) in both cases, only the resonances related to the fundamental modes $\mathrm{TEM}_{0,0, \mathrm{q}}$ are visible. The transverse modes $\mathrm{TEM}_{0,1, \mathrm{q}}$ and $\mathrm{TEM}_{1,0, \mathrm{q}}$ associated to the $q^{\text {th }}$ axial mode move out at half way between the $q$ and $q+1$ modes. The $\mathrm{TEM}_{0,2, \mathrm{q}}$ and $\mathrm{TEM}_{2,0, \mathrm{q}}$ move out to coincide with the $\mathrm{TEM}_{0,0, \mathrm{q}+2}$ modes [7]. The confocal situation simulated shows a slight difference in the resonant peaks between spherical and parabolic cavity, due to the different geometries. This difference can be solved by calculating the exact equivalent radius of curvature of the parabolic reflector by using Equation (10), with $q, D$ and $f_{\mathrm{r}, \mathrm{f}}$ fixed. The simulation results indicate that, despite the difference between parabolic and spherical geometries at this wavelength, the behavior of the two cavities is similar, allowing the use of parabolic instead of spherical reflectors.

\subsection{Setup and Experiments}

The setup used for the experiments is shown in Figure $2 b$ ). The parabolic reflectors have the same dimensions of the simulated ones, with a diameter of $60 \mathrm{~cm}$. They are connected to two waveguides WR-90 where the power is coupled to the cavity through two iris of $9.5 \mathrm{~mm}$. The waveguides are then connected to a vector network analyzer (VNA) to measure the $S_{21}$ parameter. For alignment purpose, the reflectors are installed on linear stages that permit shifts with $10 \mu \mathrm{m}$ accuracy in the $x$ direction (see Figure 1). A rotation system allows movements of the reflectors in the range of $4^{\circ}$ in elevation and azimuth. In the center, a stage with foam on it, out of the Gaussian beam region, is used as sample holder. The distance $D$ is equal to 46 $\mathrm{cm}$ and calculable from the distance between two resonant peaks associated to the fundamental modes by the equation [11]:

$$
D=\frac{c}{2 \Delta f}
$$

In Equation (15) we need to take into account that the speed of the light in the medium is equal to $c=c_{0} / n$, where $c_{0}$ is the speed of the light in the vacuum and $n$ is the refractive index of the medium (air in this case).

In Figure 4, the measured $S_{21}$ of the empty cavity is shown. In the frequency range $8.5 \mathrm{GHz}-12.5 \mathrm{GHz}, 12$ resonant peaks with a spacing of $300 \mathrm{MHz}$ are present. Each resonant peak is associated to symmetric and asymmetric fundamental modes. This means that the dielectric material can be characterized in a discrete number of twelve points corresponding to 12 resonant peaks, within the range from 8.5 to $12.5 \mathrm{GHz}$. When the dielectric material is placed inside the cavity, the resonant peaks shift proportionally to the refractive index of the material to lower frequencies, with a larger shift in case of symmetric axial mode. The larger shift is probably due to the intensity of the field being maximum for symmetric modes (node at the center of the cavity) and minimum for asymmetric modes (antinode at the center of the cavity), as illustrated in Figure 5, increasing or decreasing the effect of the perturbation by the sample. Because of the difference in shifts, it is suggested to use thick samples with asymmetric modes and thin samples with symmetric modes for more accurate results [4].

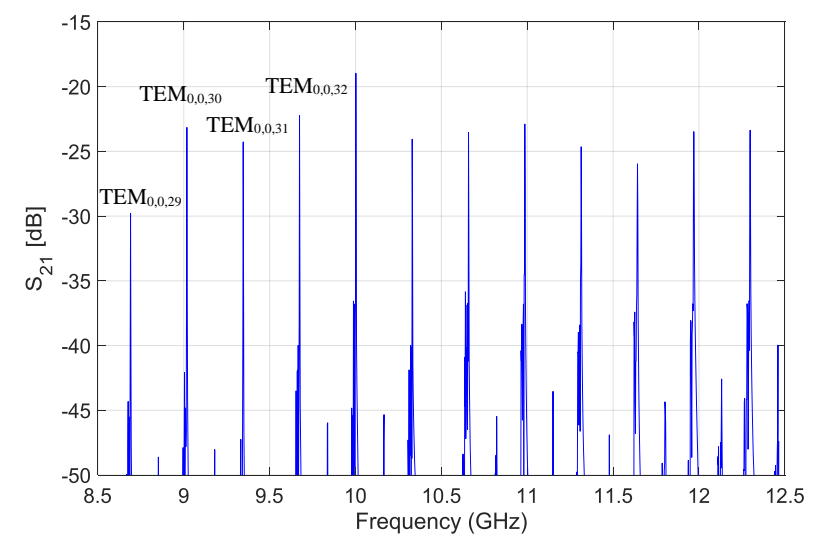

Figure 4. Resonance peaks associated to different values of $q$ in an empty cavity. 


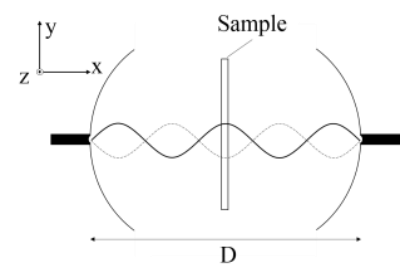

(a)

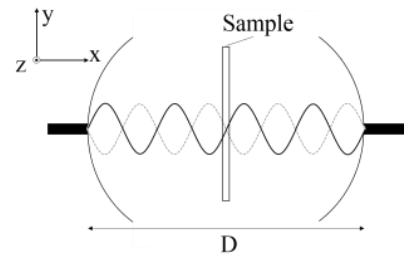

(b)
Figure 5. (a) Sketch of the resonant cavity in the case of a symmetric longitudinal resonant mode with antinode in the center of the cavity and, (b) the same cavity in the case of an antisymmetric mode with a node in the center of the cavity.

Three different samples were used: RO4003C, RO4350B and a sample of FR4. RO4003C and RO4350B materials of Rogers Corporation are hydrocarbon/ceramics laminates designed for high frequency applications, providing, as described in their datasheet, constant dielectric properties and low losses in a wide frequency range. The relative permittivity $\varepsilon_{\mathrm{r}}$, according to the datasheet, is equal to 3.55 and 3.66 respectively in the frequency range $8-40 \mathrm{GHz}$ and the loss tangent $\tan \delta$, at $10 \mathrm{GHz}$, is equal to 0.0027 and 0.0037 respectively.

Experiments were carried out on samples with a size of $120 \times 90 \mathrm{~mm}$ and with a thickness equal to $1.52 \mathrm{~mm}$ for the Roger materials and $100 \times 60 \mathrm{~mm}$ with thickness equal to $1.35 \mathrm{~mm}$ for the FR4 material.

Care must be taken to ensure that the dimension of the samples is large enough to neglect electric fields scattering from the edges out of the resonator increasing the losses of the open resonator [2]. As a rule of thumb, in order to get appropriate results, it is proposed to use samples with a minimum size equal to 2.5 times the diameter of the Gaussian beam [12]. Results of the measured $S_{21}$ are shown in Figure 6, where we can see how the shift related to the symmetric mode located at $9.018 \mathrm{GHz}$ is more significant than the shift of the asymmetric mode located at $9.346 \mathrm{GHz}$. This property of the resonant cavity is also used as reference for the alignment of the sample at the center of the cavity.

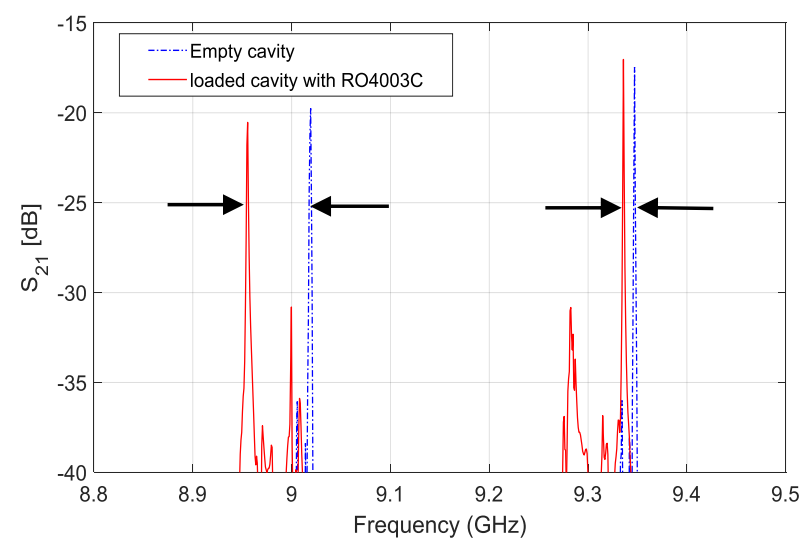

Figure 6. Shifts of the symmetric (at $9.018 \mathrm{GHz}$ ) and antisymmetric (at $9.346 \mathrm{GHz}$ ) modes of the cavity loaded with a RO4003C sample.
Moving the sample inside the cavity in the axial direction ( $x$ axis), the peak will shift to the higher frequency if the sample moves to the antinode, to the lower frequency if the sample is moving to the node. The peak reaches its minimum frequency when it is at the node of the symmetric longitudinal mode and, therefore, at the center of the cavity.

The new resonant frequencies are now used for the extraction of the relative permittivity. By entering the new frequency value in Equation (2) for symmetric and in Equation (3) for antisymmetric modes, it is possible to calculate the refractive index $n$ and thus the relative permittivity $\varepsilon_{\mathrm{r}}$.

For our experiments, because of the small sample thickness, only symmetric modes are used for the characterization. Results of the calculated relative permittivity $\varepsilon_{\mathrm{r}}$ are shown in Table 1.

\begin{tabular}{|c|c|c|c|c|c|c|}
\hline $\mathrm{f}(\mathrm{GHz})$ & 9.018 & 9.67 & 10.33 & 10.99 & 11.64 & 12.3 \\
\hline$\varepsilon_{\mathrm{r}(\mathrm{RO} 4003 \mathrm{C})}$ & 3.549 & 3.539 & 3.571 & 3.555 & 3.519 & 3.549 \\
\hline$\varepsilon_{\mathrm{r}(\mathrm{RO} 4350 \mathrm{~B})}$ & 3.717 & 3.668 & 3.693 & 3.686 & 3.686 & 3.668 \\
\hline$\varepsilon_{\mathrm{r}(\mathrm{FR} 4)}$ & 4.422 & 4.36 & 4.341 & 4.302 & 4.249 & 4.244 \\
\hline
\end{tabular}

Table 1. Measured relative permittivity $\left(\varepsilon_{\mathrm{r}}\right)$ for RO4003C, RO4350B, FR 4 with $\mathrm{t}=1.52 \mathrm{~mm}, \mathrm{t}=1.52 \mathrm{~mm}$ and $\mathrm{t}=1.35 \mathrm{~mm}$ respectively, at the resonant frequency of the symmetric modes.

To further validate the measurement results, two standard methods for dielectric material characterization are used: the two microstrip line method and the two-layer stripline method [5]. The first method is based on the characterization of the dielectric material, used as a substrate of two microstrip transmission line with different lengths, from the measure of the phase difference. The phase difference is then used to calculate the effective permittivity ( $\left.\varepsilon_{\text {eff }}\right)$ and, consequently, $\varepsilon_{\mathrm{r}}$. In the second method, instead, the material to be characterized is clamped on the top of a microstrip transmission line with accurately known characteristics. $\varepsilon_{\text {eff }}$ is calculated from the phase difference between the uncovered microstrip line and the phase of the microstrip line with the sample clamped on the top of it. Figure 7 shows the comparison between the results obtained by using the parabolic resonator and the two microstrip line methods for characterizing the RO4003C sample. We can see how the results are within the range of $2 \%$ and, in some points, overlapping.

For the validation of the other two samples, the two-layer stripline method is used. The samples are clamped on the top of the microstrip with RO4003C as substrate previously characterized. The measurements results for the RO4350B and FR4 samples are shown in Figure 7 and Figure 8 respectively. We can see a maximum deviation in $\varepsilon_{\mathrm{r}}$ of 0.18 compared to the parabolic resonator at the frequency of $10.33 \mathrm{GHz}$. We assume the airgap between the sample and the microstrip introduced an error in the final measurements.

\begin{tabular}{|l|l|l|l|}
\hline $\mathrm{f}=9.4028 \mathrm{GHz}$ & $\mathrm{RO} 4003 \mathrm{C}$ & $\mathrm{RO} 4350 \mathrm{~B}$ & $\mathrm{FR} 4$ \\
\hline $\tan \delta$ & 0.0034 & 0.0048 & 0.027 \\
\hline
\end{tabular}

Table 2. Measured values of the loss tangent $(\tan \delta)$ at 9.4028 $\mathrm{GHz}$ for the RO4003C, RO4350B, FR4 materials. 
Calculation of loss tangent requires the measurement of the quality factor of the empty cavity $Q_{0}$ and the quality factor of the loaded cavity $Q_{1}$, as previously mentioned in Section 2 . In order to increase and optimize the quality factor of the resonator, a modification to the distance between the reflectors is applied. $Q_{0}$, at the distance of $45.45 \mathrm{~cm}$ and at the frequency of $9.4063 \mathrm{GHz}$, is approximately equal to 26000 . Table 2 shows the results for the same samples previously used for the calculation of the relative permittivity, pointing out how the two Roger materials have less losses than the FR4 sample. For the $\tan \delta$ measurements, the comparison with the two nonresonant methods used above is not provided, since the transmission/reflection methods cannot give accurate results, especially for low loss samples [1].

\subsection{Uncertainty of permittivity measurement}

Accuracy of the relative permittivity measurement, looking at Equation (2) and (3), is determined by the uncertainties propagation of the resonant frequency, the distance between the reflectors and the thickness of the sample.

The combined uncertainty on the relative permittivity $\left(\Delta \varepsilon_{r}\right)$ can be approximated, for an open resonator, by propagating the individual uncertainties to the permittivity and combining them [13] using the root-sum-of-squares:

$$
\Delta \varepsilon_{r}=\sqrt{\left(\frac{\delta \varepsilon_{r}}{\delta t} \Delta t\right)^{2}+\left(\frac{\delta \varepsilon_{r}}{\delta D} \Delta D\right)^{2}+\left(\frac{\delta \varepsilon_{r}}{\delta f_{r}} \Delta f_{r}\right)^{2}}
$$

where $\Delta t$ is uncertainty of the material thickness, $\Delta D$ the uncertainty of the distance between the reflectors and $\Delta f_{r}$ the uncertainty of the resonant frequency. Equation (16) assumes that all the uncertainties are uncorrelated and all of them have Gaussian distribution. Since we do not have information about their distributions, we use the Type B Evaluation of Standard Uncertainty [14]. For the frequency, a rectangular distribution is a more reasonable model, since the probability that the value lies in that interval is $100 \%$ and equiprobable to be close to the center or at the edge of the interval itself. If we divide half of its uncertainty interval by $\sqrt{3}$, we get the standard uncertainty of the frequency, which corresponds to a probability of $68.27 \%$ that the value lies in that interval. But if it is known that the values near to the center have higher probability, like in the case of thickness and distance, then a Gaussian distribution may be a better model. For their conversion in standard uncertainty, we need to divide the uncertainty interval by two. Now that we have all standard uncertainties of the separate uncertainty contributions, we may use the root-sum-of-squares to combine them [14].

The resolution of the measured frequency is $100 \mathrm{kHz}$, obtained with 4000 point in a frequency span of $400 \mathrm{MHz}$. For the distance, an uncertainty of $\pm 10 \mu \mathrm{m}$ is given by the stages on which the reflectors are assembled and the sample thickness is measured with an accuracy approximately $\pm 5 \mu \mathrm{m}$. Converted into standard uncertainty, these intervals become $\pm 28.87 \mathrm{KHz}$ for the frequency, $\pm 10 \mu \mathrm{m}$ for the distance and $\pm 5 \mu \mathrm{m}$ for the thickness. $\Delta t$ represents the uncertainty whit the highest contribution in the calculation of the $\varepsilon_{\mathrm{r}}$ standard uncertainty.

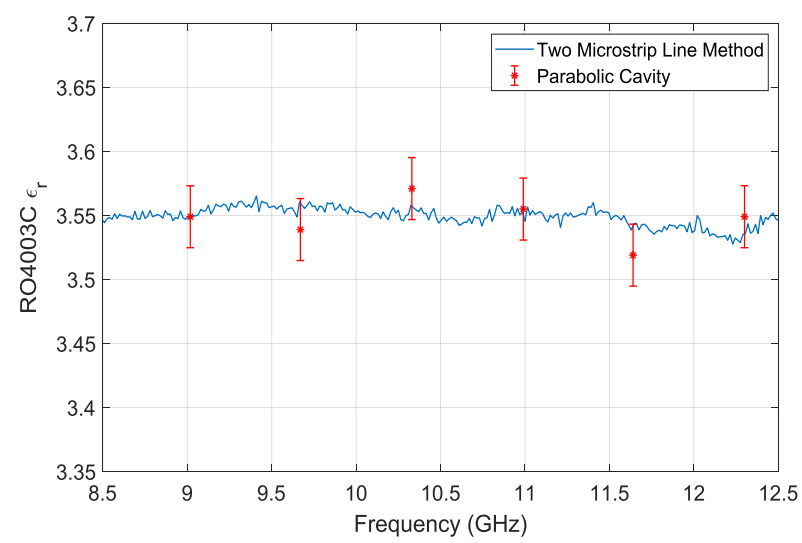

Figure 7. RO4003C relative permittivity measured by the two microstrip line methods and the parabolic resonator, for which the Standard uncertainty $\left(\Delta \varepsilon_{\mathrm{r}}\right)$ is also shown using errorbars

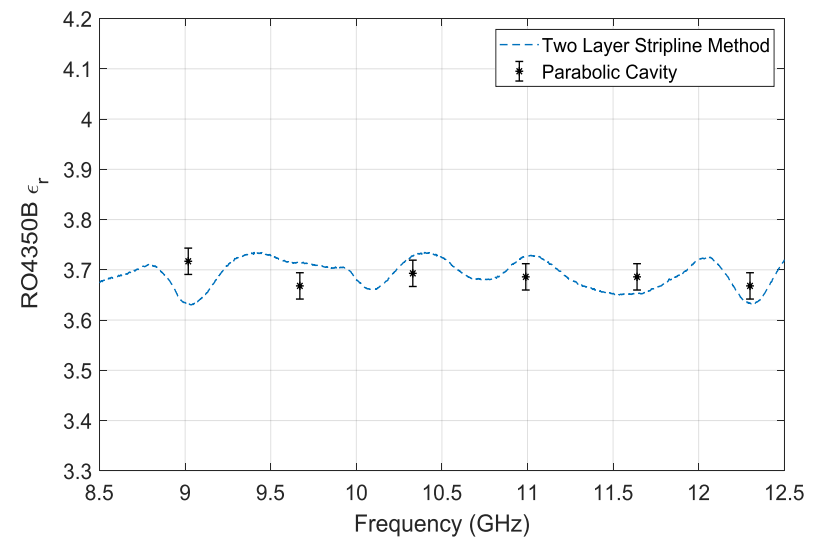

Figure 8 . RO4350B relative permittivity measured by the two layer stripline line methods and the parabolic resonator, for which the Standard uncertainty $\left(\Delta \varepsilon_{\mathrm{r}}\right)$ is also shown using errorbars.

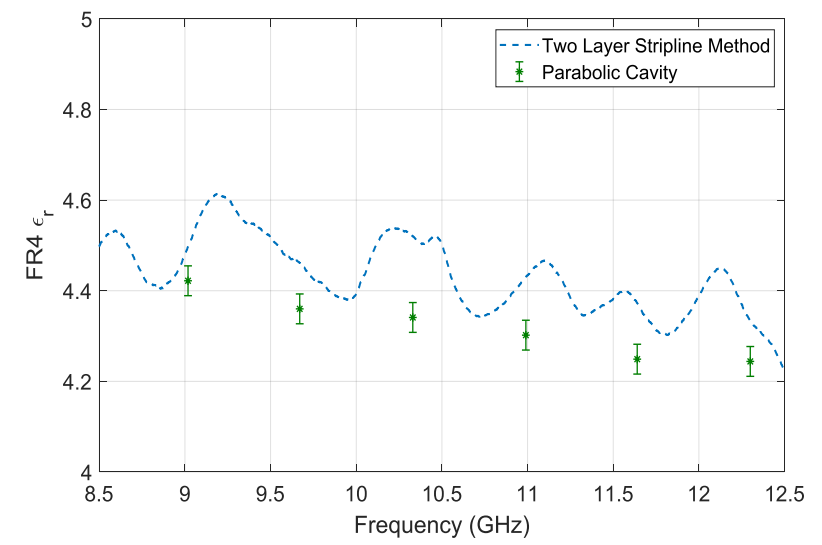

Figure 9. FR4 relative permittivity measured by the two layer stripline line methods and the parabolic resonator, for which the Standard uncertainty $\left(\Delta \varepsilon_{\mathrm{r}}\right)$ is also shown using errorbars.

The standard uncertainties of $\varepsilon_{\mathrm{r}}$, corresponding to a $68.27 \%$ confidence interval, calculated by Equation (16) at the frequency of $9.018 \mathrm{GHz}$ (approximately the same at the other frequencies), are listed in Table 3 . It shows that the uncertainties in our measurements setup are approximately $0.7 \%$ and, for each sample, they are illustrated in Figure 7, 8, 9 
using errorbars. The Standard uncertainty can be converted in Expanded uncertainty by multiplying it by 1.96 , which defines an interval having level of confidence of approximately $95 \%$ [14]. This would result in an expanded uncertainty of \pm 0.0474 , \pm 0.0513 and \pm 0.0645 for $\mathrm{RO} 4003 \mathrm{C}$, RO4835B and FR4 respectively, with a $95 \%$ confidence interval.

These results are obtained by using our instrumentation available in the laboratory, but it can certainly be improved using more accurate instruments, for example, in the measurement of the sample thickness.

\begin{tabular}{|c|c|c|c|}
\hline $\begin{array}{c}\text { Standard } \\
\text { Uncertainties }\end{array}$ & RO4003C & RO4835B & FR4 \\
\hline$\Delta t(\mu \mathrm{m})$ & & & \\
$\Delta D(\mu \mathrm{m})$ & \pm 10 & \pm 5 & \pm 5 \\
$\Delta f_{\mathrm{r}}(\mathrm{KHz})$ & \pm 28.87 & \pm 28.87 & \pm 28.87 \\
\hline$\Delta \varepsilon_{\mathrm{r}}(\mathrm{p}=68.27 \%)$ & \pm 0.0242 & \pm 0.0262 & \pm 0.0329 \\
\hline
\end{tabular}

Table 3. $\varepsilon_{\mathrm{r}}$ Standard Uncertainty calculated from the uncertainties propagation of thickness, resonant frequency and distance at the frequency of $9.018 \mathrm{GHz}$.

\section{Discussion}

The results provided above indicate that the open resonator technique, using parabolic reflectors, can produce accurate results. The further validation, performed by using two standard methods (two microstrip transmission line and twolayer stripline), shows similar results especially in the case of the two microstrip line method. The comparison with two layer stripline method shows a difference that, we believe, is due to the airgap between sample and microstrip which introduced an error in the measurements.

The low cost of the cavity (made by two parabolic reflectors easily fundable in the market) makes this setup a good solution to characterize materials, with the advantage to be nondestructive method (compared to, for example, the two microstrip line technique showed in Section 3), which in many cases is desirable for engineered materials companies.

\section{Conclusion}

In this paper a low-cost Fabry-Perot open cavity resonator for material characterization is presented. The well-known Gaussian beam theory is applied to an open cavity resonator composed of two parabolic reflectors, normally used for satellite television broadcasting. A comparison between spherical and parabolic reflectors is carried out in simulation, allowing the use of parabolic reflectors and thus reducing the cost for the design of an open resonator. The test of the setup is performed by using three different materials, and material positioning accuracy is improved using a new technique. An uncertainty analysis, based on our instrumentation, is performed. It shows that the Standard uncertainty of the measurement system is approximately $0.7 \%$, which is achieved in part thanks to the improvement of the sample positioning inside the cavity. The thickness uncertainty has the most relevant impact, but this can be improved in case of more accurate instruments for the measurement of the material thickness.

\section{References}

[1] L. F. (Lin F. Chen, Microwave electronics: measurement and materials characterization. Wiley, 2004.

[2] R. G. Jones, "Precise dielectric measurements at 35 $\mathrm{GHz}$ using an open microwave resonator," Proc. Inst. Electr. Eng., vol. 123, no. 4, p. 285, 1976.

[3] W. F. P. Chan and B. Chambers, "Measurement of Non-Planar Dielectric Samples Using an Open Resonator," in 1987 IEEE MTT-S International Microwave Symposium Digest, 1987, pp. 273-276.

[4] A. L. Cullen and P. K. Yu, "The Accurate Measurement of Permittivity by Means of an Open Resonator," Proc. R. Soc. A Math. Phys. Eng. Sci., vol. 325, no. 1563, pp. 493-509, Dec. 1971.

[5] N. K. Das, S. M. Voda, and D. M. Pozar, "Two Methods for the Measurement of Substrate Dielectric Constant," IEEE Trans. Microw. Theory Tech., vol. 35, no. 7, pp. 636-642, Jul. 1987.

[6] T. Li, H. Kogelnik, and T. Li, "Laser Beams and Resonators.pdf," IEEE Procedure vol. 54, no. 10, pp. 1312-1329, 1966.

[7] A. E. Siegman, Lasers. University Science Books, 1986.

[8] P. K. Yu and A. L. Cullen, "Measurement of Permittivity by Means of an Open Resonator. I. Theoretical," Proc. R. Soc. A Math. Phys. Eng. Sci., vol. 380, no. 1778, pp. 49-71, Mar. 1982.

[9] A. L. Cullen and P. K. Yu, "Complex Source-Point Theory of the Electromagnetic Open Resonator," Proc. R. Soc. A Math. Phys. Eng. Sci., vol. 366, no. 1725, pp. 155-171, Jun. 1979.

[10] "CST MICROWAVE STUDIO 3D EM Simulation Software." [Online]. Available: http://www.cst.com/products/cstmws. [Accessed: 18Jul-2018].

[11] K. F. Renk, "Basics of Laser Physics," 2017, pp. 1742.

[12] R. Felbecker, W. Keusgen, and M. Peter, "Estimation of permitivitty and loss tangent of high frequency materials in the millimeter wave band using a hemispherical open resonator," 2011 IEEE Int. Conf. Microwaves, Commun. Antennas Electron. Syst. COMCAS 2011, 2011.

[13] I. Hughes and T. Hase, "Measurements and their Uncertainties: A practical guide to modern error analysis."

[14] B. N. Taylor and C. E. Kuyatt, "Guidelines for Evaluating and Expressing the Uncertainty of NIST Measurement Results," 1994. 